\title{
Partial and Analog Feedback for MISO Precoding Systems
}

\author{
Didier Le Ruyet ${ }^{\dagger}$, Berna Özbek * \\ $\dagger$ Electronics and Communications Laboratory, CNAM, 292 rue Saint Martin, 75141, Paris, France \\ e-mail: leruyet@cnam.fr \\ * Electrical and Electronics Engineering Department, Izmir Institute of Technology, Urla, 35430, Izmir, Turkey \\ e-mail: bernaozbek@iyte.edu.tr
}

\begin{abstract}
We consider a multiple input single output antenna system with a feedback link. While most of the previous works have considered perfect feedback channels, in this paper, we evaluate the impact of a noisy feedback channel on the quality and the performance of the transmission. We compare the finite rate feedback scheme with the analog feedback schemes where the channel parameters are transmitted without quantization over the uplink channel. Two analog feedback schemes are considered : the feedback of the channel vector and the feedback of the normalized channel vector. We provide bounds on the performance and give numerical results. We have shown that at low to medium uplink SNR the analog feedback of the normalized channel vector outperforms the analog feedback of the channel vector. Depending on the range of uplink SNR, quantized feedback can perform poorly compared to analog feedback. ${ }^{1} 2$
\end{abstract}

\section{INTRODUCTION}

Multiple antenna systems are playing an increasing role in wireless communications. When channel state information (CSI) is available at the transmitter, the potential gain increases considerably. Among the possible techniques, transmit beamforming or linear precoding has been recognized as an interesting solution to improve the performance of multiple antenna systems. In time division duplex (TDD) systems, assuming that the duplexing time delay is lower than the coherence time, it is possible to learn the uplink channel without transmission of the CSI. However, on frequency division duplex (FDD), due to the phase difference between the uplink and downlink channel it is generally not possible to directly estimate the uplink channel. Then, the terminal must estimate the CSI and then transmit it to the base station using the feedback channel.

A first solution is to directly transmit the unquantized precoding vector or the unquantized channel vector. It can be shown that this solution achieves the minimum mean square error (MMSE) distortion in the Shannon sense. Analog feedback has been described in [4] and recently studied in multi user wireless systems in [5].

Due to the limited bandwidth of the feedback channel another solution is to quantize the channel coefficients or the precoding vector before transmission over the finite rate link.

\footnotetext{
${ }^{1}$ The work of Didier Le Ruyet is supported by the European Eureka+Pidea SMART project.

${ }^{2}$ The work of Berna Özbek is supported by the FP6 IYTE Wireless Project.
}

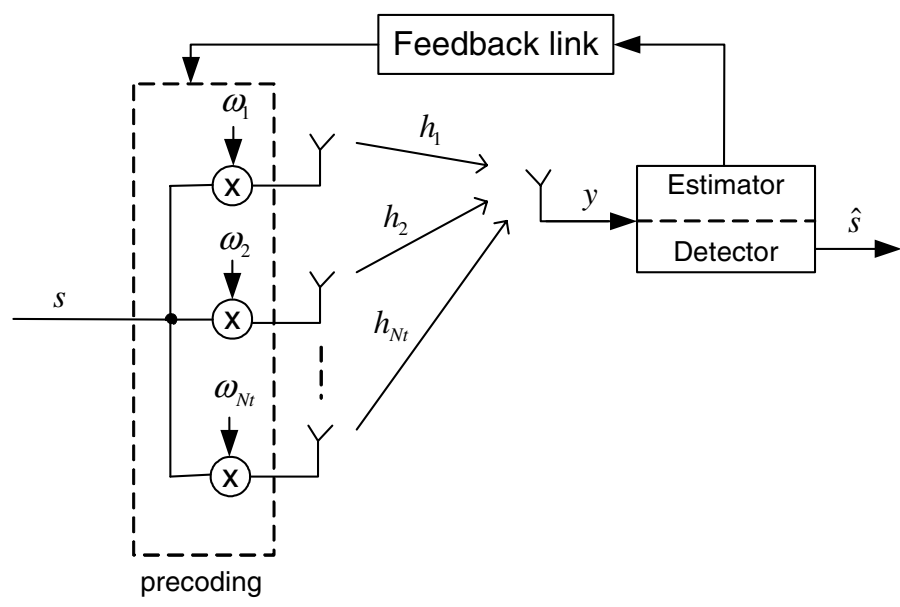

Fig. 1. communication system

In [1], the Lloyd algorithm was suggested for the design of the beamforming vector codebook. [2] and [3] showed that the codebook should be constructed by minimizing the maximum inner product between any two beamforming vectors in the codebook.

In this paper, we compare the performance of analog and quantized feedback over a noisy feedback channel. We consider the downlink channels as independent and identically distributed (i.i.d.).

The system model is described in the next section. Then, in sections 3 and 4, we evaluate analytically the distortion function considering i.i.d. downlink channels. Simulation results are presented in section 5 and conclusions are drawn in section 6.

\section{SySTEM MODEL}

We consider a single user communication system employing transmit beamforming and receive combining with $N_{t}$ transmit antennas and a single receive antenna. This communication system is presented in Figure 1.

The baseband input output discrete relationship for a given complex transmitted symbol $s$ is represented by:

$$
y=\mathbf{h w} s+n
$$




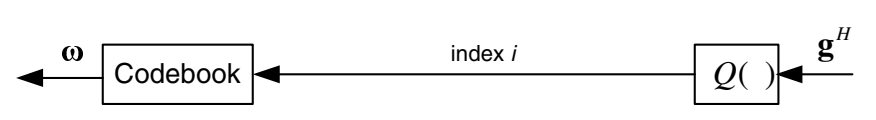

(a)
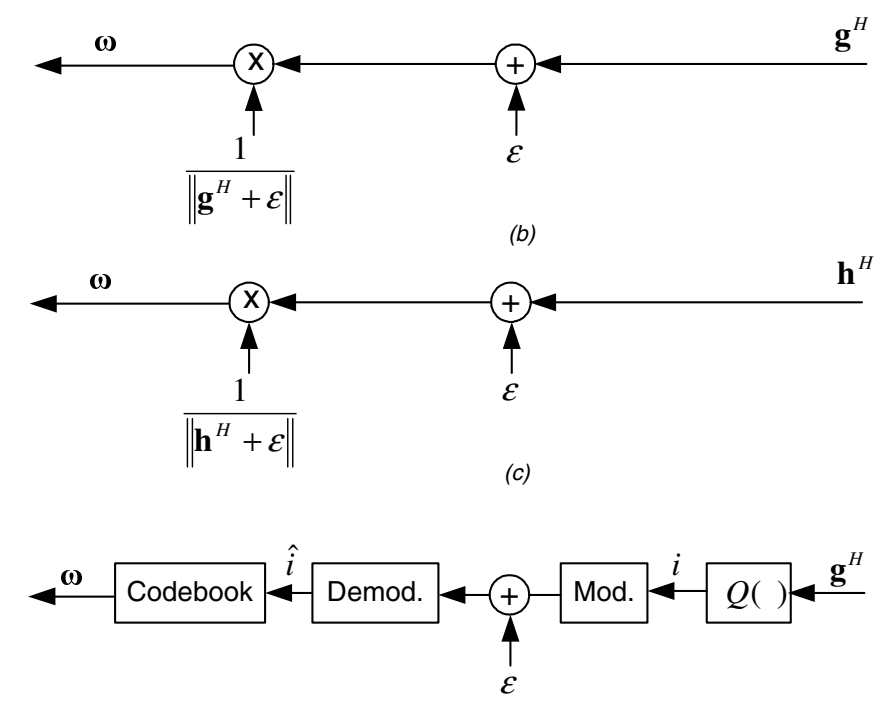

(d)

Fig. 2. different feedback links : (a) quantized feedback (b) noisy analog feedback of the normalized channel vector $\mathbf{g}(\mathrm{c})$ noisy analog feedback of the channel vector $\mathbf{h}(\mathrm{d})$ noisy quantized feedback

where $\mathbf{h}=\left[\begin{array}{llll}h_{1} & h_{2} & \ldots & h_{N_{t}}\end{array}\right]$ is the channel vector and $\mathbf{w}=$ $\left[\begin{array}{lllll}w_{1} & w_{2} & \ldots & w_{N_{t}}\end{array}\right]^{T}$ is the precoding vector.

The instantaneous signal-to-noise ratio (SNR) is given by:

$$
\gamma=\frac{E_{S}}{N_{0}}|\mathbf{h w}|^{2}
$$

We define the normalized channel vector:

$$
\mathrm{g}=\frac{\mathbf{h}}{\|\mathbf{h}\|}
$$

In this paper, we consider different feedback links as shown in Figure II. Since we focus on the impact of the feedback link on the performance, we will consider that the channel vector $\mathbf{h}$ has been perfectly estimated by the terminal.

Since analog feedback use analog modulation, we avoid the problem associated to the quantization of the channel. However, compared to digital modulation, the dynamic range is much larger. In this paper, we will not consider the possible RF transmitter problems related to this large dynamic range. We will consider two analog feedback schemes :

- analog transmission of the channel vector $\mathbf{h}$

- analog transmission of the normalized channel vector $g$

In the quantized feedback scheme, the vector $\mathbf{w}$ is taken from a set of $N=2^{B}$ vectors where $B$ is the number of feedback bits. The construction of the set of the precoding vectors $\mathbf{W}=\left[\mathbf{w}_{1}, \mathbf{w}_{2}, \ldots, \mathbf{w}_{N}\right]$ can be seen as a line packing problem [2][3]. For i.i.d. channels, we have:

$$
\begin{aligned}
\mathbf{W}_{\text {opt }} & =\min _{\mathbf{W} \in \mathcal{C}^{N_{t} \times N}} \max _{1 \leq i<j \leq N}\left|\mathbf{w}_{i}^{H} \mathbf{w}_{j}\right| \\
& =\max _{\mathbf{W} \in \mathcal{C}^{N_{t} \times N}} \min _{1 \leq i<j \leq N} d\left(\mathbf{w}_{i}, \mathbf{w}_{j}\right)
\end{aligned}
$$

where

$$
d\left(\mathbf{w}_{i}, \mathbf{w}_{j}\right)=\sqrt{1-\left|\mathbf{w}_{i}^{H} \mathbf{w}_{j}\right|^{2}}
$$

$\mathbf{w}_{i}$ can be seen as the coordinates of a point situated at the surface of an hypersphere with a unit radius centered at the origin.

The instantaneous SNR can also be written as :

$$
\gamma=\frac{E_{S}}{N_{0}}\|\mathbf{h}\|^{2}(1-Z)
$$

where

$$
\begin{aligned}
Z & =\min _{i} d^{2}\left(\mathbf{g}^{H}, \mathbf{w}_{i}\right) \\
& =\min _{i}\left(1-\left|\mathbf{g} \mathbf{w}_{i}\right|^{2}\right)
\end{aligned}
$$

$Z$ is a random variable within the interval $[0,1]$. Denote $p(z)$ and $F(z)$ as the probability density function and the cumulated distribution function of $Z$. In the next section, we evaluate the symbol error rate (SER) from $p(z)$.

\section{AVERAGE SER APPROXIMATION FOR QUANTIZED FEEDBACK}

For the Rayleigh fading channel, the SER can be evaluated using the Craig's formula [6]:

$$
\operatorname{SER}(\gamma)=\frac{1}{\pi} \int_{0}^{\frac{(M-1) \pi}{M}} \exp \left(-\frac{g_{P S K} \gamma}{\sin ^{2} \theta}\right) d \theta
$$

where $M$ is the constellation size and $g_{P S K}=\sin ^{2}(\pi / M)$ is the constellation dependent term.

The average SER can be calculated by averaging over all the possible instantaneous SNR $\gamma$ [7]:

$$
\begin{aligned}
\overline{S E R} & =\int_{\gamma=0}^{\infty} \operatorname{SER}(\gamma) p(\gamma) d \gamma \\
& =\frac{1}{\pi} \int_{0}^{\frac{(M-1) \pi}{M}} \int_{0}^{1}\left(1+\frac{g_{P S K}(1-z) \frac{E_{s}}{N_{0}}}{\sin ^{2} \theta}\right)^{-N_{t}} p(z) d z d \theta
\end{aligned}
$$

In [3] [7], the authors have introduced an upper bound on $F(z)$ for quantized feedback scheme assuming that the regions associated to each codeword do not overlap :

$$
F(z) \leq \tilde{F}(z)\left\{\begin{array}{lll}
N z^{N_{t}-1} & \text { if } & 0 \leq z<N^{-\frac{1}{N_{t}-1}} \\
1 & \text { if } & z \geq N^{-\frac{1}{N_{t}-1}}
\end{array}\right.
$$




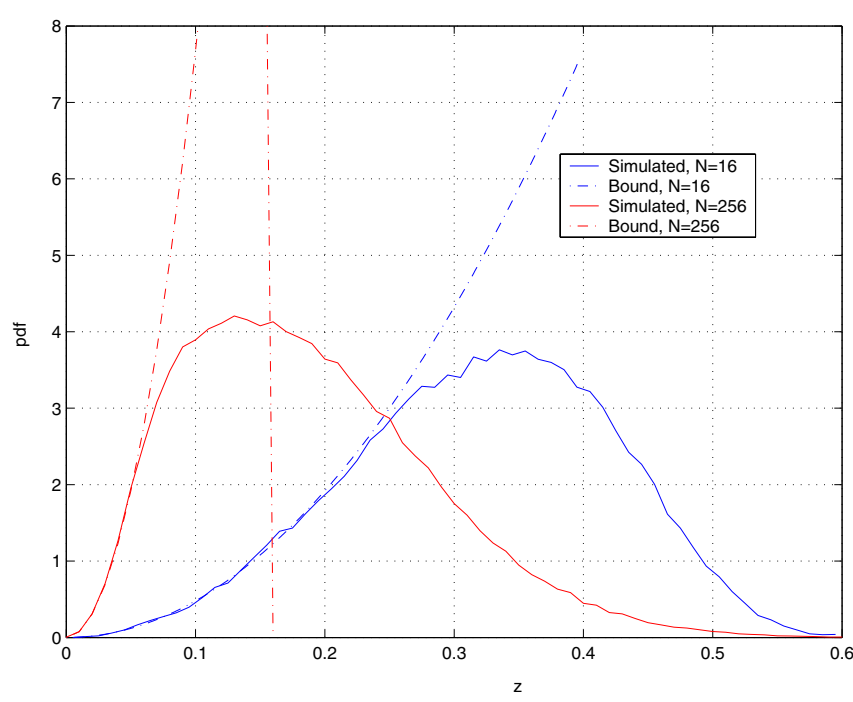

Fig. 3. probability density function $p(z)$ and $\tilde{p}(z)$ for $N_{t}=4$ and $N=$ 16,256 for the quantized feedback.

and the associated power density function (pdf) $\tilde{p}(z)$ :

$$
\tilde{p}(z)=\left\{\begin{array}{lll}
N\left(N_{t}-1\right) z^{N_{t}-2} & \text { if } & 0 \leq z<N^{-\frac{1}{N_{t}-1}} \\
0 & \text { if } & z \geq N^{-\frac{1}{N_{t}-1}}
\end{array}\right.
$$

This pdf is given in Figure III for $N_{t}=4$ and $N=16,256$. In this Figure, we also present the pdf obtained using the best packing codebook given in [8] for $N=16$ and a codebook based on Fast Fourier Transform matrices for $N=256$ using a randomly incomplete search [9]. We can observe that there is a noticeable difference between the bound and the simulated pdf.

Finally, we can obtain a lower bound on the average SER by replacing $p(z)$ by (11) in equation (9) [7]. We have

$$
\overline{S E R}_{l b}=\frac{1}{\pi} \int_{0}^{\frac{(M-1) \pi}{M}} A(\theta) d \theta
$$

with

$$
A(\theta)=\left(1+\frac{g_{P S K} \bar{\gamma}}{\sin ^{2} \theta}\right)^{-1}\left[1+\left[1-\left(\frac{1}{N}\right)^{\frac{1}{N_{t}-1}}\right] \frac{g_{P S K} \bar{\gamma}}{\sin ^{2} \theta}\right]^{1-N_{t}}
$$

While the pdf are quite different, In figure 7 we can see that for $N=16$ the lower bound is very close to the average simulated SER obtained using the codebook presented previously (0.2 dB shift only).

When the feedback channel is noisy, the decoded codeword index can be different from the original one. We introduce a random variable $Y$ taking into account the possible transmission errors.

$$
Y=1-\left|\mathbf{g w}_{j}\right|^{2}
$$

where

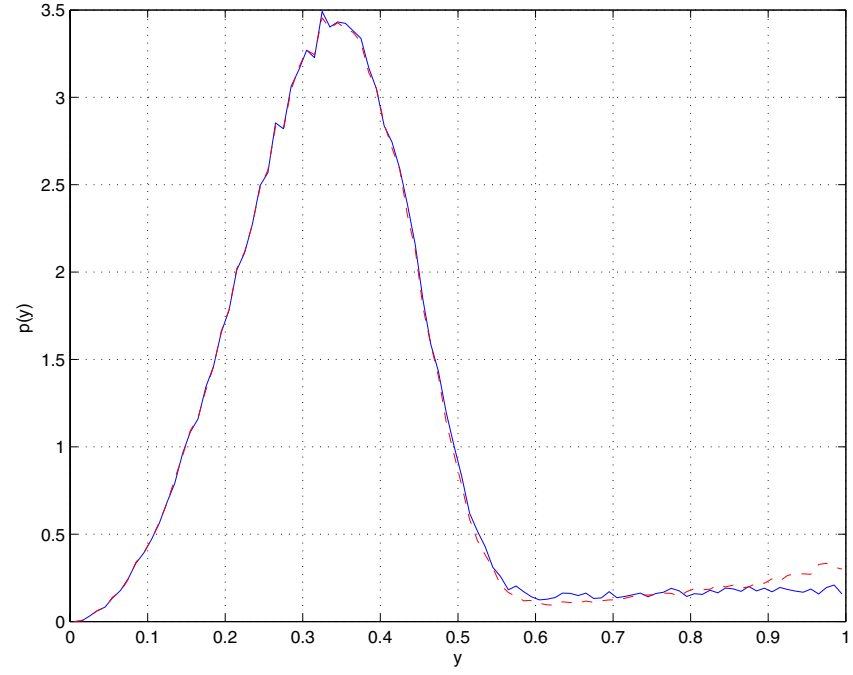

Fig. 4. probability density function $p(y)$ for the quantized feedback.

$\begin{cases}j=\arg \min _{i}\left(1-\left|\mathbf{g w}_{j}\right|^{2}\right) & \text { if } \text { no transmission error } \\ j \neq \arg \min _{i}\left(1-\left|\mathbf{g} \mathbf{w}_{j}\right|^{2}\right) & \text { if } \text { transmission error }\end{cases}$

The pdf of $Y$ is obtained by the association of two pdf $p_{C}(y)$ and $p_{E}(y)$ corresponding respectively to the non transmission/transmission error case. $p_{C}(y)$ is the same pdf than the previous pdf $p(z)$. We have :

$$
p(y)=(1-C E R) p_{C}(y)+C E R p_{E}(y)
$$

The codeword error rate (CER) depends on the chosen modulation.

In Figure 4, we give the pdf $p(y)$ obtained for $N_{t}=4$, $N=16$ codewords and $\left(E_{s} / N_{0}\right)_{U L}=4 d B$ using MonteCarlo simulations. The 4 bits have been encoded using 4 BPSK symbols. We can observe the impact of the noise on this pdf. As shown in Figure 4, by optimizing the mapping between the codewords and the transmitted symbols, it is possible to slightly improve the performance.

The pdf $p_{E}(y)$ is difficult to evaluate analytically. However, for a given normalized channel vector $\mathbf{g}$, asymptotically the received vectors $\mathbf{w}_{j}$ are uniformly distributed over the unitary radius hypersphere. Consequently we can find a SER lower bound by approximating $p_{E}(z)$ as a uniform pdf.

From (9), the SER is expressed as :

$$
\begin{aligned}
\overline{S E R} & =\frac{1}{\pi} \int_{0}^{\frac{(M-1) \pi}{M}}\left[\int_{0}^{1}\left(1+\frac{g_{P S K}(1-y) \frac{E_{s}}{N_{0}}}{\sin ^{2} \theta}\right)^{-N_{t}}\right. \\
& \times(1-C E R) p_{C}(y) d y \\
+ & \left.\int_{0}^{1}\left(1+\frac{g_{P S K}(1-y) \frac{E_{s}}{N_{0}}}{\sin ^{2} \theta}\right)^{-N_{t}} C E R p_{E}(y) d y\right] d \theta
\end{aligned}
$$

From this result and using the previous lower bound we obtained the modified SER lower bound as follows : 
$\overline{S E R}_{l b}=\frac{1}{\pi} \int_{0}^{\frac{(M-1) \pi}{M}}\left[A(\theta)(1-C E R)+\frac{1}{N_{t}-1} C E R\right]_{(18)} d \theta$

\section{SER PERFORMANCE FOR ANALOG FEEDBACK}

In this section, we consider two analog feedback schemes: the normalized channel vector or precoding vector feedback and the non-normalized channel vector feedback. In both scheme, we assume that the mobile has performed a perfect estimation of the channel vector $\mathbf{h}$.

In the first version, the normalized channel vector $\mathrm{g}$ is transmitted over the noisy feedback channel. The received vector is given by:

$$
\mathbf{w}^{\prime}=\mathbf{g}^{H}+\epsilon
$$

After normalization we have:

$$
\mathbf{w}=\frac{\mathbf{w}^{\prime}}{\left\|\mathbf{w}^{\prime}\right\|}
$$

Each element of the noisy vector $\epsilon$ is a zero mean complex gaussian noise with variance $\sigma_{U L}^{2}=\frac{\left(N_{0}\right)_{U L}}{2}$ per dimension.

The precoding vector is applied to the transmitted vector. The received signal $y$ is given by:

$$
y=\mathbf{h w} s+n
$$

As previously, $Z$ is a random variable within the interval $[0,1]$. We have the following relation:

$$
\begin{aligned}
Z & =1-|\mathbf{g} \mathbf{w}|^{2} \\
& =1-\left|\mathbf{g} \frac{\mathbf{g}^{\mathbf{H}}+\epsilon}{\left\|\mathbf{g}^{\mathbf{H}}+\epsilon\right\|}\right|^{2} \\
& =1-\left|\frac{1+\mathbf{g} \epsilon}{\left\|\mathbf{g}^{\mathbf{H}}+\epsilon\right\|}\right|^{2} \\
& =1-\frac{1+2 \Re(\mathbf{g} \epsilon)+|\mathbf{g} \epsilon|^{2}}{1+2 \Re(\mathbf{g} \epsilon)+\|\epsilon\|^{2}}
\end{aligned}
$$

In the second version, the channel vector $\mathbf{h}$ is transmitted over the noisy feedback channel. The received vector is given by :

$$
\mathbf{w}^{\prime}=\mathbf{h}^{H}+\epsilon
$$

We have :

$$
\mathbf{w}=\frac{\mathbf{h}^{H}+\epsilon}{\left\|\mathbf{h}^{H}+\epsilon\right\|}
$$

We have the following relation :

$$
\begin{aligned}
Z & =1-|\mathbf{g w}|^{2} \\
& =1-\left|\mathbf{g} \frac{\mathbf{h}^{\mathbf{H}}+\epsilon}{\left\|\mathbf{h}^{\mathbf{H}}+\epsilon\right\|}\right|^{2} \\
& =1-\frac{\|h\|^{2}+2\|h\| \Re(\mathbf{g} \epsilon)+|\mathbf{g} \epsilon|^{2}}{\|h\|^{2}+2\|h\| \Re(\mathbf{g} \epsilon)+\|\epsilon\|^{2}}
\end{aligned}
$$

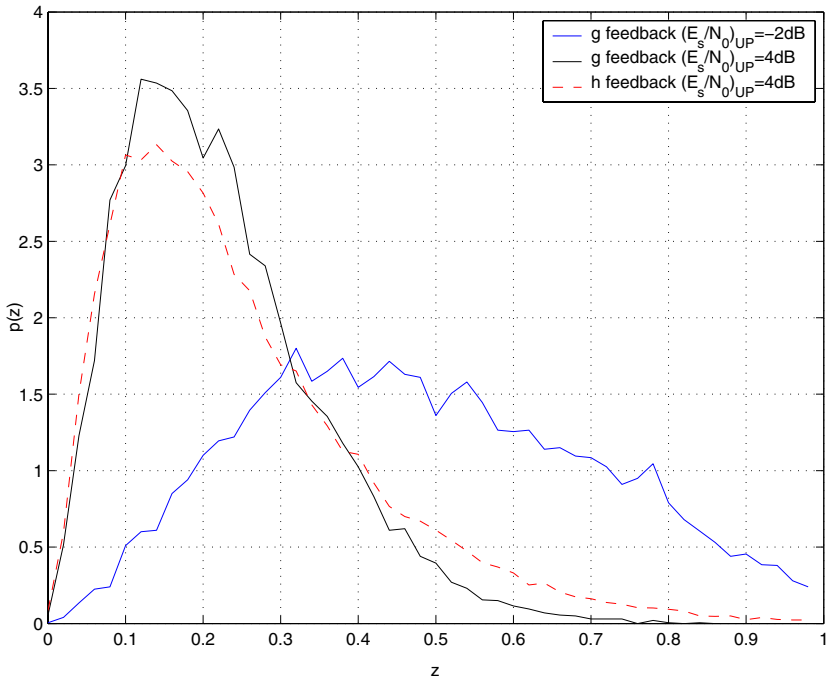

Fig. 5. probability density function $p(z)$ for the analog feedback.

Since it is difficult to compare the expressions (22) and (26) we will evaluate the pdf of $Z$ using Monte-Carlo simulations.

The pdf $p(z)$ are given in Figure 5 for $N_{t}=4$ for the analog feedback of $\mathbf{h}$ and $\left(E_{s} / N_{0}\right)_{U L}=4 d B\left(E_{s}=1, \sigma_{U L}^{2}=0.2\right)$ and for the analog feedback of $\mathbf{w}$ and $\left(E_{s} / N_{0}\right)_{U L}=4 d B$ $\left(E_{s}=1 / N_{t}, \sigma_{U L}^{2}=0.05\right)$ and $\left(E_{s} / N_{0}\right)_{U L}=-2 d B\left(\sigma_{U L}^{2}=\right.$ $0.2)$.

From these results and using the equation (9), we can predict that an analog feedback of the normalized channel vector $\mathrm{g}$ give better performances than the analog feedback of the channel vector $\mathbf{h}$ since the tail of $p(z)$ is longer for the feedback of $\mathbf{h}$. While the feedback of $\mathbf{h}$ achieves the same MMSE distortion as a scheme that optimally quantizes and encodes the CSI in the Shannon sense [4], this scheme is not optimal from the overall performance point of view.

This result can be explained since the constraint imposed to the norm of $\mathbf{g}$ allow us to eliminate some energy of the uplink noise compared to the other scheme. Consequently $Z$ is on average lower using the normalized channel vector feedback.

\section{ON TIME CORRELATED CHANNEL}

The channel vectors are often time correlated and the base station can exploit this correlation in order to reduce the distorsion.

When analog feedback of the channel vector $\mathbf{h}$ or $\mathbf{g}$ is performed, a Kalman filter can be implemented to track the channel vector. Using the Jakes model, the elements of the channel vector $\mathbf{h}$ can be easily modelled using an AR model since the Doppler spectrum is frequency limited. On the other hand for the normalized channel $\mathbf{g}$ due to the norm constraint, it is possible to exploit the dependance between the elements. Further study must be carry on in order to evaluate the impact of this problem on the overall performance. When quantized feedback is performed, it is much more difficult to track the channel since the state space model is strongly non linear and 


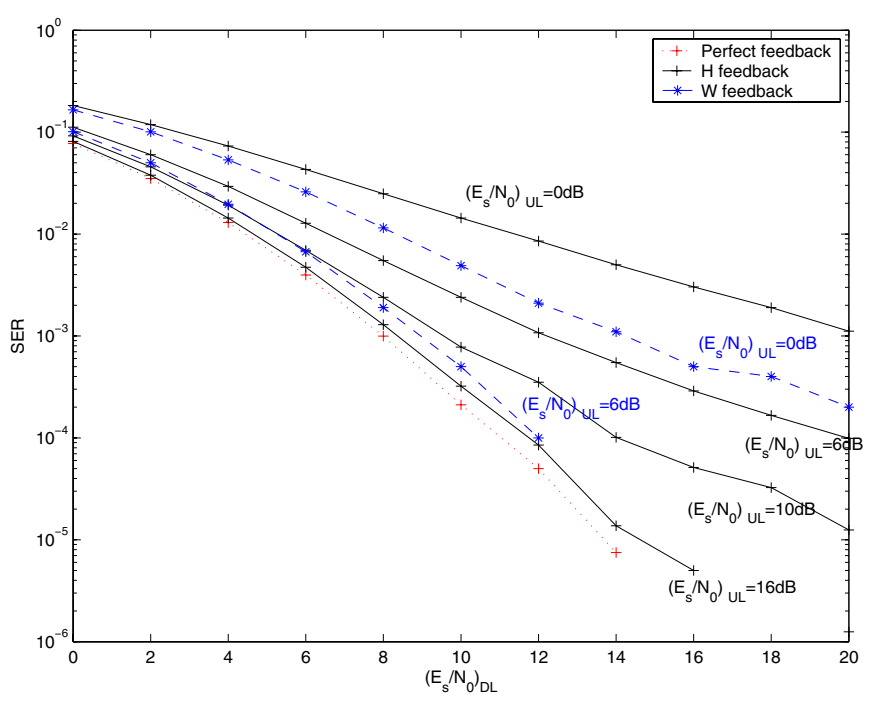

Fig. 6. $S E R=f\left(E_{S} / N_{0}\right)_{D L}$ for the analog feedback schemes.

information is only available at each transition between the codewords. Different solutions such as particle filtering are possible in order to exploit these information to reduce the distorsion.

\section{Simulation Results}

In this section we consider the different feedback schemes for $N_{T}=4$ and i.i.d. channels.

In order to fairly compare all the different schemes, we have fixed the same average transmit energy per symbol. While for the $\mathbf{g}$ feedback scheme the energy corresponding to the transmission of the vector $\mathbf{g}$ is a constant (since $\| \mathbf{g}||=1$ ), at the symbol level, the transmitted signal is gaussian with the same variance than for the $\mathbf{h}$ feedback scheme.

In figure 6, we give the performance $S E R=f\left(E_{S} / N_{0}\right)_{D L}$ of the $\mathbf{h}$ and $\mathbf{g}$ feedback schemes for different $f\left(E_{S} / N_{0}\right)_{U L}$. As shown previously the $\mathbf{g}$ feedback scheme outperforms the h feedback scheme.

In figure 7, we give the performance $S E R=f\left(E_{S} / N_{0}\right)_{D L}$ of the quantized feedback scheme for different $f\left(E_{S} / N_{0}\right)_{U L}$. The number of codewords is $N=16$ and the modulation is BPSK (4 symbols are needed to transmit one codeword index). Compared to the analog feedback schemes, we can observe a floor effect on the $S E R$ depending on the uplink noise power. We also give the curves obtained using the lower bound (18) for different $\left(E_{S} / N_{0}\right)_{U L}$. We can see from the curves that the lower bounds are quite tight.

Of course, it is possible to decrease the floor effect by adding an error correcting code (for example using 4 QPSK symbols and a Reed-Muller $(8,4)$ code to transmit one codeword index).

In figure 8 , we compare the performance of the analog and partial feedback schemes for $f\left(E_{S} / N_{0}\right)_{D L}=6 d B$. For all the schemes, 4 symbols are needed to transmit one codeword index. As shown previously, the $\mathbf{g}$ feedback scheme outperforms the other feedback schemes. While the quantized

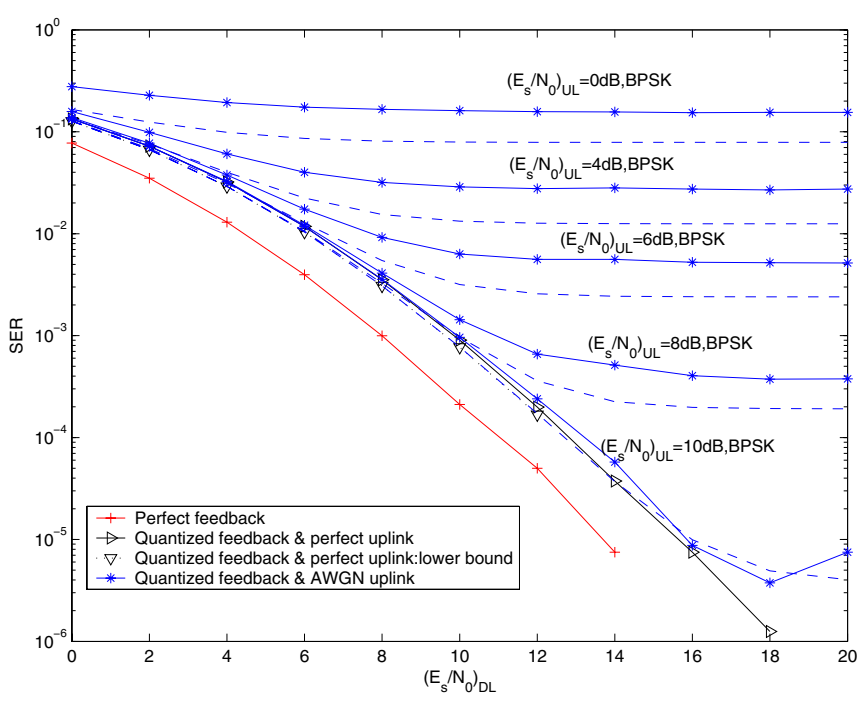

Fig. 7. $S E R=f\left(E_{S} / N_{0}\right)_{D L}$ for the quantized feedback schemes.

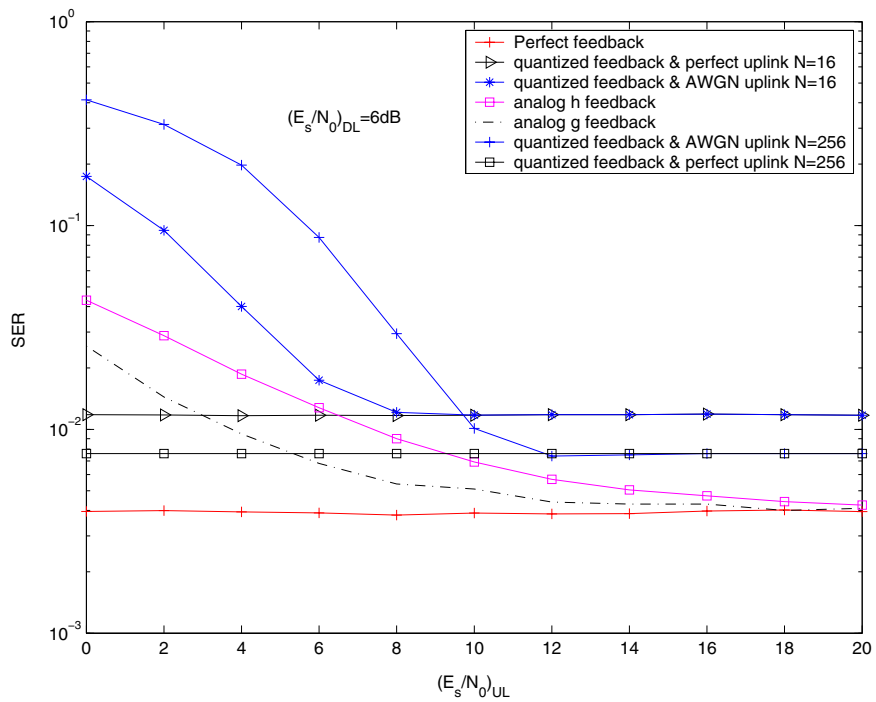

Fig. 8. Comparison of all feedback schemes with $S E R=f\left(E_{S} / N_{0}\right)_{U L}$.

precoding scheme with $N=256$ gives better performance than with $N=16$, since the quantization error is lower, at low $f\left(E_{S} / N_{0}\right)_{U L}$ this scheme is more sensitive to transmission errors.

\section{CONCLUSIONS}

In this paper, we have compared different feedback schemes for i.i.d. channels. We have shown that at low to medium SNR the analog feedback of normalized channel vector $\mathbf{g}$ gives better performance than the analog feedback of the channel vector $\mathbf{h}$. While the increase of the number of codewords in the finite rate feedback reduces the distortion on $\mathbf{w}$, for the same number of symbols per codeword, it is also more sensitive to uplink noise. As shown in the simulation section, depending on the range of uplink SNR, quantized feedback can perform 
poorly compared to analog feedback.

A further study will be the impact of correlated channels and the non linearity on the performance of such schemes.

\section{REFERENCES}

[1] A. Narula, M. J. Lopez, M. D. Trott, G. W. Wornell, "Efficient use of side information in multiple antenna data transmission over fading channels" in IEEE Journal on selected areas in communications, pp.1423-1436, oct. 1998.

[2] D. J. Love, R. W. Heath and T. Strohmer, "Grassmanian beamforming for multiple input multi output wireless systems" in IEEE Trans. on Information Theory, vol. 49-10, pp.2735-2747, oct. 2003.

[3] K. K. Mukkavilli, A. Sabarwal, E. Erkip, B. Aazhang, "On beamforming with finite rate feedback in multiple antenna systems" in IEEE Trans. on Information Theory, vol. 49-10, pp.2562-2579, oct. 2003.

[4] M. Gaspar, R. Rimoldi, M. Vetterli, "To code, or not to code: lossy source channel communication revisited" in IEEE Trans. on Information Theory, vol. 49, pp.1147-1158, May 2003.

[5] T. L. Marzetta, B. M. Hochwald, "Fast transfer of channel state information in wireless systems" in IEEE Trans. on Information Theory, vol. 54-4, pp.1268-1278, April 2006.

[6] M. K. Simon, M. S. Alouini, "A unified approach to the performance analysis of digital communication over generalized fading channels" in Proceedings of the IEEE, vol. 86-9, pp.1860-1877, sept. 1998.

[7] S. Zhou, Z. Wang, G. Giannakis, "Performance analysis for transmitbeamforming with finite rate feedback" in conf. on Information Sciences and Systems CISS, Princeton, USA, march 2004.

[8] "http://dynamo.ecn.purdue.edu/ djlove/grass.html"

[9] B. M. Hochwald, T. L. Marzetta, T. L. Richardson, W. Sweldens, R. Urbanke, "Systematic design of unitary space-time constellations" in IEEE Trans. on Information Theory, vol. 46, pp.1962-1973, Sept. 2000. 